\title{
Charge transfer at very high pressure in $\mathrm{NiO}$
}

\author{
Abhay Shukla, ${ }^{1}$ * Jean-Pascal Rueff, ${ }^{2}$ James Badro, ${ }^{3}$ Gyorgy Vanko, ${ }^{1}$ Aleksi Mattila, ${ }^{4}$ F. M. F de Groot, ${ }^{5}$ and Francesco Sette ${ }^{1}$ \\ ${ }^{1}$ European Synchrotron Radiation Facility, Boite Postale 220, F-38043 Grenoble, France \\ ${ }^{2}$ Laboratoire de Chimie Physique, CNRS, F-75231 Paris, France \\ ${ }^{3}$ Laboratoire de Minéralogie-Cristallographie, CNRS, F-75252 Paris, France \\ ${ }^{4}$ Division of X-ray Physics, Department of Physical Sciences, POB 64, University of Helsinki, FIN-00014, Finland \\ ${ }^{5}$ Department of Inorganic Chemistry and Catalysis, Utrecht University, Sorbonnelaan 16, 3584 CA Utrecht, The Netherlands
}

(Received 23 September 2002; revised manuscript received 4 December 2002; published 7 February 2003)

\begin{abstract}
We use resonant inelastic x-ray scattering to study the electronic structure of nickel oxide, the prototype charge-transfer insulator, as a function of pressure. At ambient pressure, we observe spectral features due to the charge-transfer excitation and the Coulomb correlation energy which progressively smear up to a pressure of $100 \mathrm{GPa}$. These changes are interpreted as due to increased dispersion of the concerned electronic bands. This opens new perspectives both for the study of electronic structure of correlated materials and for high-pressure research such as a deeper understanding of metal-insulator transitions, as well as the study of deep-earth chemistry.
\end{abstract}

DOI: 10.1103/PhysRevB.67.081101

PACS number(s): $62.50 .+\mathrm{p}, 71.70 . \mathrm{Ch}, 78.70 . \mathrm{En}$

Since the early observations of de Boer and Verwey, ${ }^{1}$ challenging accepted ideas by showing that partially filled bands could be insulators, the field of strongly correlated electrons has grown rapidly with new materials showing surprising properties. The mechanics of the metal to insulator transition with the variation of a given parameter (for example temperature or pressure) is central to this field. Nickel oxide is the prototype charge-transfer insulator and as such has been widely studied. It is now well established that though the $U_{d-d}$ correlation energy is relatively high in this compound, the band gap is of the charge-transfer type that is primarily $\mathrm{O} 2 p$ to Ni $3 d$ character. $^{2}$ This gap has been measured to be about $4 \mathrm{eV}$ in magnitude. ${ }^{3}$ Several calculations of the electronic structure of $\mathrm{NiO}$ exist as this has proved to be a good testing ground for theories due to the influence of strong correlations. In fact, only explicit inclusion of the effects of this strong correlation in sophisticated band-structure calculations could bring these to predict the correct band gap and excitation spectra for this compound. ${ }^{4}$ Local cluster models, however, were reasonably successful in predicting them, presumably because they dealt with the effects of correlation in a more direct way. ${ }^{5}$

As argued in the well-known pioneering work by Mott, ${ }^{6}$ an important parameter that can be varied better understanding of the electronic structure of strongly correlated transition-metal compounds is density, and therefore pressure. However, the variation of pressure either rules out the use of spectroscopic methods or imposes severe constraints on many of them. In this first use of resonant inelastic x-ray scattering (RIXS) under ultrahigh pressure, we measure charge-transfer excitations in $\mathrm{NiO}$ up to $1 \mathrm{Mbar}$. In the coherent second-order RIXS process, an incoming photon is absorbed with the system transiting to an intermediate state influenced by the strong core-hole potential. The choice of the incident energy, near an absorption edge of the system, provides a means of choosing particular intermediate states of interest. The system then decays to an excited state (giving rise to inelastic structure) or back to the ground state (resulting in an elastic line) with the emission of a photon.
The number of electrons is conserved and the excitation spectrum is measured directly. RIXS has been successfully used $^{7}$ to study the electronic structure of Mott insulators. It is also an ideal probe for high-pressure studies, since x-rays can penetrate sample environment, can be focused to small sizes, and can be tuned to a given energy ensuring resonant enhancement of the cross section.

The experiment was performed at beamline ID16 at the European Synchrotron Radiation Facility. The experimental setup was that of a Rowland circle spectrometer using a spherically focusing, elastically bent $\mathrm{Si}(551)$ analyzer and a Peltier-cooled Si-diode detector. The scattering plane was horizontal. Undulator radiation monochromatized by a cryogenically cooled $\mathrm{Si}(111)$ channel-cut monochromator with a bandwidth of $1.4 \mathrm{eV}$ was focused by a toroidal mirror. The incident-beam intensity was monitored by a Si-diode detector. The sample (polycrystalline powder from Aldrich Chemicals with a nominal $\mathrm{Ni}$ content of $76 \%$ corresponding to a stoichiometry of $\mathrm{Ni}_{0.97} \mathrm{O}$ ) was loaded in a symmetrical Mao-Bell cell with $300 \mu \mathrm{m}$ culet diamonds using a 5-mmdiameter high-strength $\mathrm{Be}$ gasket. At the energies used (8.35 $\mathrm{keV}$, around the Ni K edge) and for a $90^{\circ}$ scattering geometry, the X-ray transparent Be gasket was a critical part of the experiment. No pressure medium was used permitting us to maximize sample size, the price to pay being nonhydrostaticity. The pressure was determined using ruby fluorescence and diffraction, and the gradient was evaluated. $\mathrm{X}$ rays were incident on the sample through the gasket and slits were used to define the spot size on the sample to $30 \mu \mathrm{m}$ (vertical) $\times 110 \mu \mathrm{m}$ (horizontal). This slit size was maintained throughout the experiment as the sample size never became smaller. The measured flux on the sample was 1.3 $\times 10^{12}$ photons/s. The total resolution as measured from the full width at half maximum of the peak due to quasielastic scattering was $1.5 \mathrm{eV}$.

In an earlier study on $\mathrm{NiO}$, Kao et al. ${ }^{8}$ showed that the RIXS signal measured at a scattering angle of $30^{\circ}$ with the momentum transfer along the [001] direction consists of two peaks, centered around 4.9 and $7.8 \mathrm{eV}$. Our experiment dif- 


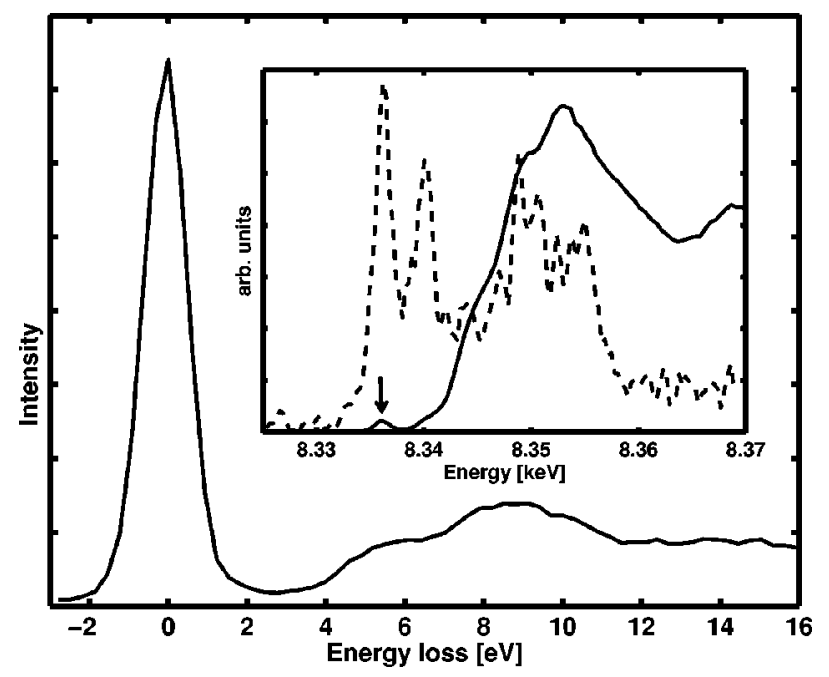

FIG. 1. Resonant inelastic x-ray scattering from polycrystalline $\mathrm{NiO}$ at ambient pressure with the incident energy tuned to the quadrupolar prepeak in the absorption spectrum. Two structures corresponding to energy losses of $5.3 \mathrm{eV}$ and $8.5 \mathrm{eV}$ are present. Inset: high-resolution partial fluorescence yield spectrum of the Ni K-edge absorption with an arrow showing the choice of incident energy (solid line). The dashed line is a constant-final-state scan (constant energy difference between incident and detected energy) at an energy loss of $5 \mathrm{eV}$, showing that the strongest resonance corresponds to the quadrupolar prepeak.

fers from the one of Kao et al. in the choice of several key experimental conditions. We used a polycrystalline $\mathrm{NiO}$ sample and worked at a scattering angle of $90^{\circ}$ (corresponding to a momentum transfer of $6 \AA^{-1}$ ), to eliminate the nonresonant inelastic signal from the Be gasket using the polarization of the incident radiation (linear, in the scattering plane). The absorption edge is shown in the inset of Fig. 1 along with a constant-final-state scan where a constant energy transfer is maintained while the incident energy is scanned across the edge. The resonances in this scan are due to particular intermediate states (selected by the choice of the incident energy) which decay to the same final state (selected by the choice of the energy transfer). At this high scattering angle, the strongest resonance for an energy transfer of $5 \mathrm{eV}$ is at the prepeak of the absorption spectrum, and other resonances, including the one corresponding to the peak of the absorption spectrum (used by Kao et al.) are strongly suppressed. We tuned our incident energy to this resonance with the following two benefits. The prepeak is associated with quadrupolar transitions (favored at large scattering angles) and the lowest-energy excited states relevant to the electronic properties of $\mathrm{NiO}$ are the $3 d^{n+1}$ configurations. Notably, the transition across the gap is from predominantly $\mathrm{O} 2 p$ states to $\mathrm{Ni} 3 d$ states. Second since the incident energy is below the edge, absorption is low ensuring sizable penetration in the sample (the absorption length is about $40 \mu \mathrm{m}$ ).

In Fig. 1, we show our RIXS data at room pressure and temperature. We measure a broad inelastic spectral feature with a marked low-energy shoulder, peaking at $5.3 \mathrm{eV}$ and a peak centered at $8.5 \mathrm{eV}$, as determined by fits to Gaussian line shapes. The energy loss $(4.3 \mathrm{eV})$ to the edge of this

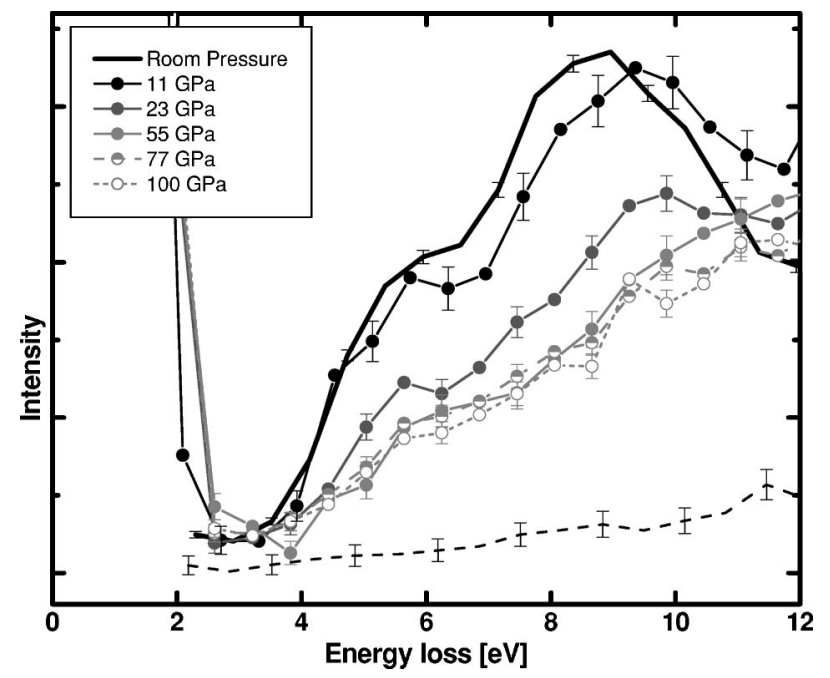

FIG. 2. RIXS data as a function of pressure. Bottom dashed line: nonresonant background. As pressure is increased the resonant intensity decreases and the 5.3 and $8.5 \mathrm{eV}$ structures smear out due to the increasing electronic bandwidth.

shoulder is the value of the charge-transfer gap in $\mathrm{NiO}, 9,3$ thus providing a direct measure of this quantity. We associate this feature to the metal-ligand transition leading to a $d^{n+1} \underline{L}$ excited state, where $d^{n}$ is the metal ground-state configuration and $\underline{L}$ is a ligand hole. We associate the peak at $8.5 \mathrm{eV}$ primarily to the metal-metal transitions leading to $d^{n+1} d^{n-1}$ excited states and thus to the correlation energy $U_{d-d}$ related to transport in the $d$ band. Given this sensitivity to the electronic structure of $\mathrm{NiO}$ in our measurement we now look for changes introduced by varying the pressure on the sample. In Fig. 2, we show the RIXS data as a function of increasing pressure up to $100 \mathrm{GPa}$. The data is normalized to the monitor intensity. The dashed line is a measurement of the nonresonant background with the incident energy at $8370 \mathrm{eV}$, well above the absorption edge. We also show for comparison arbitrarily scaled data (labeled room pressure) measured at ambient pressure outside the pressure cell. As pressure is increased, the resonant inelastic x-ray scattering intensity progressively decreases. Second, the double structure (shoulder and peak) clearly resolved at ambient and lower pressures, smears at pressures above $50 \mathrm{GPa}$ into a poorly defined line shape. By fitting the RIXS data with a sum of three Gaussians to account for the two features mentioned above as well as the intensity at higher energies, this trend can be better understood. The 5.3-eV feature does not shift appreciably as pressure is increased, while its width doubles from 2 to $4 \mathrm{eV}$. The $8.5-\mathrm{eV}$ peak shifts to above $10 \mathrm{eV}$ up to 50 $\mathrm{GPa}$ and then remains constant, while its width also increases from 4 to $5 \mathrm{eV}$. As pressure is increased, electronic bands increasingly disperse which means that the sharp resonances seen at lower pressures become ill defined as seen by the increasing width of the peaks. In particular, overlap with the anions increases since the lattice parameter changes by about $10 \%$ at $100 \mathrm{GPa}$. Calculations suggest that the shape of the electronic density of states does not change much with pressure but the density of states decreases uniformly and bandwidth increases. ${ }^{10}$ This is compatible with the behavior 

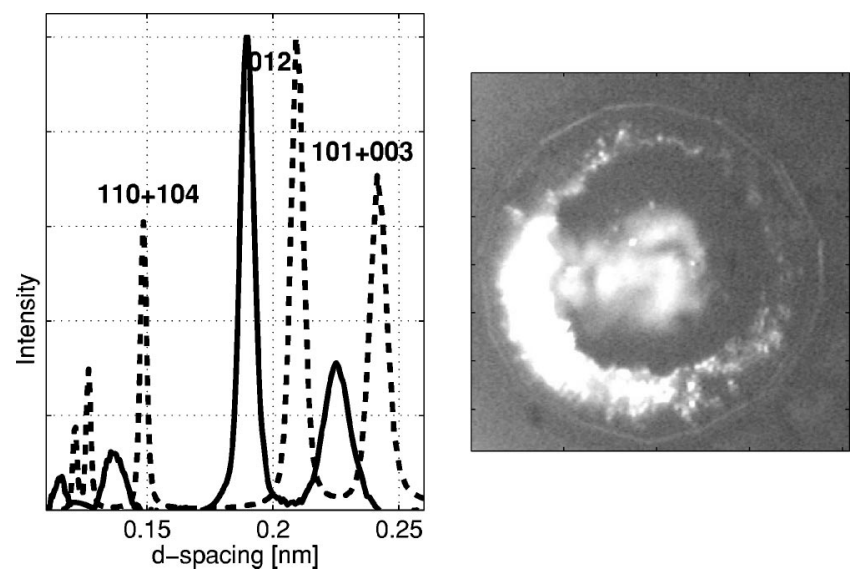

FIG. 3. Left panel. Diffraction patterns of the sample at room pressure (dashed line) and $100 \mathrm{GPa}$ (solid line) with some principal peaks indexed. Overlapping peaks become broader at higher pressures indicating lattice distortions due to nonhydrostaticity. From the refinement and comparison with the ruby fluorescence, we estimate a pressure gradient of $\pm 10 \mathrm{GPa}$ at $100 \mathrm{GPa}$. The change in lattice spacing confirms the pressure value indicated by the ruby fluorescence. Right panel. A photograph of the sample at $100 \mathrm{GPa}$ through a diamond anvil showing the bright outer ring which is the Be gasket, a dark inner ring corresponding to the outer parts of the sample, and a shiny inner core that assumes this luster only at the highest pressures. When pressure is released, the luster disappears.

of the 5.3-eV peak, which increases in width without appreciable change in position. It is the increase in width or the increased dispersion that reduces the value of the chargetransfer gap. The behavior of the $8.5-\mathrm{eV}$ peak would suggest an initial increase in the $d-d$ Coulomb interaction with pressure. Though this is unexpected, since screening increases with pressure, we remark that it is limited to lower pressures. Finally, the observed trends suggest that a metal-insulator transition would happen due to the closing of the chargetransfer gap as seen in other charge-transfer insulators at lower pressures. ${ }^{11}$ It is important to ascertain that this change in electronic structure is associated with band widening due to increased overlap or due to changes in the crystal field consequent to structural change. $\mathrm{NiO}$ is not known to undergo structural phase transitions till well above $100 \mathrm{GPa}$, as a recent study has shown. ${ }^{12}$ We performed powder diffraction with high-energy $(93 \mathrm{keV}) \mathrm{x}$-rays at ambient pressure and at $100 \mathrm{GPa}$. The diffraction patterns are shown in Fig. $3(\mathrm{a})$, and are in excellent agreement with the data of Eto et al. ${ }^{12}$

Above a pressure of about $50 \mathrm{GPa}$, we do not observe a significant change in the RIXS signal. However at higher pressures, changes do occur as observed visually on the sample and as shown by the Ni-K near-edge structure. At 77 GPa, the sample observed through a microscope showed signs of metallic luster, which became clearly defined at 100 GPa. A photograph [Fig. 3(b)] of the sample at $100 \mathrm{GPa}$ shows that the central core reflects light in contrast with the dark ring around it. This luster disappeared when pressure was removed. Preliminary optical reflectivity measurements on the 100-GPa sample show a shift of the $4 \mathrm{eV}$ ambient

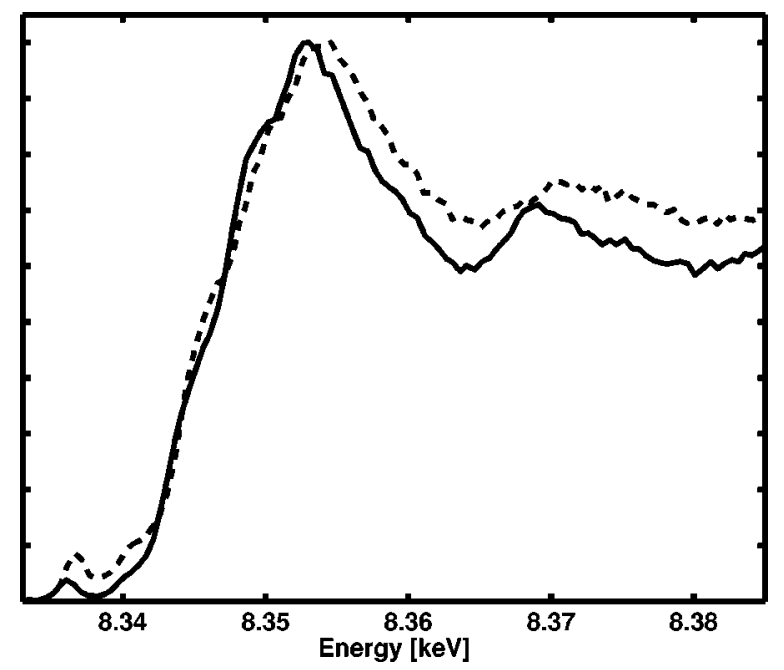

FIG. 4. Change in the absorption spectrum from ambient pressure (solid line) to $100 \mathrm{GPa}$ (dashed line) due to decrease in the lattice constant and increase in bandwidth with pressure. The $\mathrm{Ni}$ $\mathrm{K}$-edge spectrum was measured using partial fluorescence yield with the spectrometer set on the $\mathrm{K} \beta$ peak while the incident energy was varied across the edge.

pressure peak in $\mathrm{NiO}$ to $3 \mathrm{eV}$ and a tail extending into the gap causing the metallic luster. ${ }^{13}$ It is also possible that the measured pressure gradient could cause a local lowering of symmetry and precipitate changes in electronic structure at pressures lower than expected for the $\mathrm{NiO}$ structure. This question could be settled through further measurements using a pressure medium such as $\mathrm{He}$ and through calculations that would help to quantify the effect of pressure, as well as that of a gradient on the RIXS signal.

In Fig. 4, we show the Ni K-edge measured by using the spectrometer at ambient pressure and at $100 \mathrm{GPa}$, by monitoring the partial $(\mathrm{K} \beta)$ fluorescence yield, with intensity normalized to the peak (white line). With this technique, the core-hole lifetime broadening is considerably reduced resulting in sharper spectral features. ${ }^{14}$ Structure in the edge is broadened at high pressure due to increasing bandwidth and the peak shifts by $1.3 \mathrm{eV}$ to higher energies, as expected. Further the prepeak region gains in intensity probably due to an increase in hybridization between the local $3 d$ and $4 p$ bands.

In conclusion, we have measured resonant inelastic losses in $\mathrm{NiO}$ as a function of pressure up to a megabar. We find a significant change that can be traced back to the modification of electronic structure induced by pressure, namely, increase in electronic bandwidth leading to a decrease of the chargetransfer gap. The charge-transfer and correlation energies do not change appreciably in the measured range. These measurements should stimulate further work both experimental and theoretical, including measurements where the pressure gradient is minimized and calculations of RIXS in $\mathrm{NiO}$ as a function of pressure. These results point to the potential of this method to investigate matter under high pressure as well changes in electronic structure related to metal-insulator transitions with temperature or doping, especially in highly correlated materials. They might also help to address some of the fundamental questions in the field of deep-earth 
geochemistry, such as the depletion of nickel in earth's lower mantle.

We thank K. Syassen for discussions and reflectivity measurements, R. Verbeni for the preparation of the analyzer crystal, H. K. Mao for providing us with the gasket material, and V. Honkimäki and M. Mezouar for help in powder diffraction. We acknowledge discussions with A. Kotani and K. Hämäläinen. A. Mattila was supported by the Academy of Finland (Grant No. 7379/39182/40732).
*Present address: Laboratoire de Minéralogie-Cristallographie, case 115, 4 Place Jussieu, 75252, Paris Cedex 5 France.

${ }^{1}$ J.H. de Boer and E.J.W. Verwey, Proc. Phys. Soc., London, Sect. A 49, 59 (1937)

${ }^{2}$ M. Imada, A. Fujimori, and Y. Tokura, Rev. Mod. Phys. 70, 1039 (1998).

${ }^{3}$ S. Hüfner, Adv. Phys. 43, 183 (1994), and references therein.

${ }^{4}$ V.I. Ansimov, J. Zaanen, and O.K. Andersen, Phys. Rev. B 44, 943 (1991); Z. Szotek, W.M. Temmermann, and H. Winter, ibid. 47, 4029 (1993); F. Aryasetiawan and O. Gunnarsson, Phys. Rev. Lett. 74, 3221 (1995).

${ }^{5}$ A. Fujimori and F. Minami, Phys. Rev. B 29, 5225 (1984); 30, 957 (1984).

${ }^{6}$ N.F. Mott, Proc. Phys. Soc. A 62, 416 (1949).

${ }^{7}$ J.P. Hill, C.-C. Kao, W.A.L. Caliebe, M. Matsubara, A. Kotani, J.L. Peng, and R.L. Greene, Phys. Rev. Lett. 80, 4967 (1998); P.
Abbamonte, C.A. Burns, E.D. Isaacs, P.M. Platzman, L.L. Miller, S.W. Cheong, and M.V. Klein, ibid. 83, 860 (1999); M.Z. Hasan, E.D. Isaacs, Z.-X. Shen, L.L. Miller, K. Tsutsui, T. Tohyama, and S. Maekawa, Science 288, 1811 (2000).

${ }^{8}$ C.-C. Kao, W.A.L. Caliebe, J.B. Hastings, and J.-M. Gillet, Phys. Rev. B 54, 16361 (1996).

${ }^{9}$ G. Sawatzky and J.W. Allen, Phys. Rev. Lett. 53, 2339 (1984).

${ }^{10}$ R.E. Cohen, I.I. Mazin, and Donald. G. Isaak, Science 275, 654 (1997).

${ }^{11}$ P. Dufek, P. Blaha, and K. Schwarz, Phys. Rev. B 51, 4122 (1995).

${ }^{12}$ T. Eto, S. Endo, M. Imai, Y. Katayama, and T. Kikegawa, Phys. Rev. B 61, 14984 (2000).

${ }^{13} \mathrm{~K}$. Syassen (private communication).

${ }^{14}$ K. Hämäläinen, D.P. Siddons, J.B. Hastings, and L.E. Berman, Phys. Rev. Lett. 67, 2850 (1991). 